\title{
Detection of parvovirus B19 IgM by antibody capture enzyme immunoassay: receiver operating characteristic analysis
}

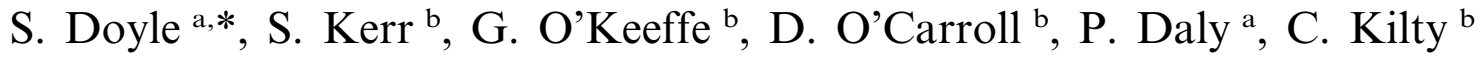 \\ a Department of Biology, National University of Ireland, Maynooth, Co. Kildare, Ireland \\ ${ }^{\mathrm{b}}$ Biotrin International, The Rise, Mount Merrion, Dublin, Ireland
}

Received 5 January 2000; received in revised form 30 June 2000; accepted 3 July 2000

\begin{abstract}
Parvovirus B19 infection can cause severe effects in high-risk groups including pregnant women and immunocompromised individuals. Although serological detection of B19 infection is commonplace, minimal information is available on the absolute performance characteristics of various tests for the detection of B19 IgM. The performance of the first parvovirus B19 IgM enzyme immunoassay to be cleared by the US Food and Drug Administration (FDA) is described. The immunoassay cut-off has been established using receiver operating characteristic (ROC) analysis giving a sensitivity and specificity of detection of 89.1 and $99.4 \%$, respectively. No cross-reactivity is observed with rubella or other viral disease IgM which cause similar symptomologies to parvovirus B19. Multi-site reproducibility studies have shown high immunoassay reproducibility with detection rates (observed/expected result) of $100 \%$ for nonreactive specimens $(N=324)$ and strongly reactive $(N=403)$, respectively. Immunoassay reproducibility ranged from 11.76 to $17.46 \%$ coefficient of variation for all reactive specimens tested $(N=12)$ whereby each specimen was assayed a total of 81 times. Parvovirus B19 IgM seroprevalence of $1 \%$ was observed in a US blood donor population $(N=399)$. In the absence of international performance criteria, this study will be of major benefit to the clinical virologist in assessing immunoassay reliability for the detection of recent infection with parvovirus B19. (C) 2000 Elsevier Science B.V. All rights reserved.
\end{abstract}

Keywords: Serology; Diagnosis; Infection; Fetus; Pregnancy; Immunocompromised

\section{Introduction}

Since its discovery in 1975, parvovirus B19 has been identified as the causative agent of a number

\footnotetext{
* Corresponding author. Tel.: + 353-1-7083858; fax: + 3531-7083845.

E-mail address: sean.doyle@may.ie (S. Doyle).
}

of clinical conditions in humans (Anderson et al., 1985; Kurtzman et al., 1987). The spectrum of symptoms caused by parvovirus B19, including rash, arthralgia and transient aplastic crisis are generally self-limiting in healthy individuals. However, serious complications due to viral infection may arise in certain populations including pregnant women, immunocompromised patients 
and individuals suffering from sickle cell disease or other blood-related disorders (Serjeant et al., 1993; Jordan, 1996; Török, 1997). In fact, exposure to parvovirus B19 during pregnancy leads to fetal loss occurring in $8-16 \%$ of infections (Mark et al., 1993; Jordan, 1996).

The parvovirus B19 capsid is composed of two structural proteins, namely VP1 $(83 \mathrm{kDa})$ and VP2 (53 kDa), with VP2 comprising approximately 95\% of total capsid protein (Ozawa and Young, 1987; Ozawa et al., 1987). Both of these proteins have been expressed in numerous prokaryotic and eukaryotic expression systems in order to assess their potential use as diagnostic reagents for parvovirus B19 infection (Morinet et al., 1989; Brown et al., 1990). Indeed, O'Neill et al. (1995) concluded that both baculovirus-derived parvoviral antigens (VP1 and VP2) were of equivalent potency with respect to antibody detection and that the superior sensitivity of detection obtained with respect to the native virus may be due to the more controlled purification conditions associated with recombinant antigen purification.

Both parvovirus B19 IgM detection and PCR detection of viral DNA are now used to detect acute or recent parvovirus B19 infection (Török et al., 1992; Cohen, 1997). In view of this, it is of significant concern that there is no requirement for serological test validation prior to use as a parvovirus B19 in vitro diagnostic in most countries. In fact, no data is actually available on the absolute performance characteristics of in vitro diagnostic assays used widely and all previous reports on the use of parvovirus B19 IgM detection systems have focused mainly on either comparative studies either between commercial kits from different manufacturers or commercial kits with in-house immunoassays. For instance, Sloots and Devine (1996) reported the relative sensitivity and specificity ranges of four commercial immunoassays ranging from $97-100$ to $81-99 \%$, respectively. Notably, these authors identified rubella IgM cross-reactivity as particularly problematic in a number of commercial immunoassay formats. In a similar study design, Tolfvenstam et al. (1996) evaluated the performance of a number of commercial immunoassays using a panel of 203 clinical specimens and again noted the propensity of some commercial parvovirus B19 IgM immunoassays to exhibit cross-reactivity with rubella, Epstein-Barr virus (EBV) and cytomegalovirus IgM. Furthermore, these researchers noted that a false positivity rate of 5\% was evident when sera from healthy pregnant women were analysed in some commercial immunoassays. In a recent study, Pickering et al. (1998) compared commercial immunoassay performance to that of an in-house immunoassay, which uses parvovirus B19 VP1 expressed in CHO cells, and concluded that the commercial assays exhibited a specificity and sensitivity ranges of 91.4-93.5 and 97.4-97.5\%, respectively. In our view, this information is of limited value to the clinical virologist in deciding which test format to use, firstly because no information is ever provided as to absolute immunoassay performance and secondly because in-house tests are rarely, if ever, subject to any external regulatory or technical scrutiny. Furthermore, none of the aforementioned studies present any information relating to immunoassay cut-off standardisation and reproducibility or, more importantly, the use and robustness of the immunoassays in routine clinical use.

Data are now described on the absolute performance characteristics of the first parvovirus B19 IgM enzyme immunoassay to be cleared by the US Food and Drug Administration (FDA) in terms of cut-off standardisation, sensitivity, specificity, antibody class specificity, immunoassay reproducibility and antibody seroprevalence.

\section{Materials and methods}

\subsection{Antigen production, purification and modification}

Parvovirus B19 recombinant VP2 capsids used for parvovirus B19 IgM detection were expressed in the baculovirus expression system using Spodoptera frugipera cells (Brown et al., 1990, 1991). Capsid purification has been previously described (Kerr et al., 1999) and the resultant 
capsid VP2 was biotinylated using $N$-hydroxysuccinimid-obiotin at a 50:1 (reagent:protein) molar ratio.

\subsection{Parvovirus B19 IgM enzyme immunoassay}

The parvovirus B19 IgM enzyme immunoassay used for this study is a mu-capture enzyme immunoassay for the detection of parvovirus B19 IgM in human serum and plasma (1/100 dilution). Microwells were pre-coated with rabbit anti-human IgM and total serum IgM was bound to the solid phase $(1 \mathrm{~h})$. Following a wash step, the biotinylated VP2 capsids were added which bound to any parvovirus B19 $\mathrm{IgM}$ present (30 $\mathrm{min}$ ). After another wash step, streptavidin-peroxidase was added which, in turn, bound to the biotinylated VP2 present (30 min). This complex was then detected by addition of tetramethyl benzidine (TMB) substrate. Sulphuric acid (1 N) addition terminated the reaction after $10 \mathrm{~min}$ and was followed by absorbance measurement at $450 \mathrm{~nm}$. Specimens with an index value (specimen/cut-off absorbance ratio) less than 0.9 and greater than 1.1 were deemed nonreactive and reactive, respectively. Specimens in the index range $0.9-1.1$ were deemed equivocal.

\subsection{Receiver operating characteristic (ROC) analysis}

ROC analysis was used to assess the clinical accuracy of the parvovirus B19 IgM enzyme immunoassay. Here, the relative sensitivity and specificity at various decision threshold values (assay cut-off values) were calculated following the evaluation of (i) a sensitivity panel from individuals with parvovirus B19 infection diagnosed clinically $(N=110)$ and (ii) a specificity panel of putative cross-reactive specimens $(N=164)$, respectively. Immunoassay sensitivity and specificity were therefore determined as follows:

1. Sensitivity: specimens $(N=110)$ used to establish the sensitivity of the parvovirus B19 IgM immunoassay, met the following criteria: (i) patients were diagnosed independently by a physician as having clinical symptoms concordant with parvovirus B19 infection and (ii) specimens were analysed using a parvovirus B19 IgM immunofluorescent assay (IFA) to evaluate IgM status, where possible. Hence, the parvovirus B19 IgM immunoassay itself was not used to classify the immune status of the subjects in accordance with accepted guidelines for ROC analysis (NCCLS, 1995). It should be noted that clinical diagnosis was taken as the primary diagnostic, thus, a specimen was deemed to be parvovirus B19 IgM reactive in the case of a positive clinical diagnosis and an nonreactive IFA test result (two specimens fell into this category).

2. Specificity: specimens from patients with disease states symptomatically analogous to parvovirus B19 infection or with putative cross-reactive potential were tested to establish the specificity of the parvovirus B19 IgM immunoassay for ROC analysis. This panel comprised 164 individual serum samples including 21 cytomegalovirus (CMV) IgM, 25 EBV IgM, 20 rubella $\operatorname{IgM}$, five rubeola IgM, five mumps IgM, five varicella zoster virus (VZV) IgM, five herpes simplex virus (HSV)-1 and $2 \mathrm{IgM}$, five parainfluenza-3 IgM, four influenza B IgM, five mycoplasma IgM, 14 toxoplasmosis IgM, five lyme disease IgM, 17 rheumatoid factor, five rheumatoid arthritis, five thyroiditis, five systemic lupus erythematosus and eight with anti-nuclear antibodies (ANA). These specimens were confirmed as mostly strong reactives for their particular condition using FDA cleared in vitro diagnostic devices except thyroiditis, rheumatoid arthritis and lupus sera. These conditions were diagnosed clinically by physicians and the patients were known to be undergoing treatment for the specific disorders at the time of the sample donation.

To classify further patients, all available specimens were also tested by a parvovirus B19 IFA which employs a different antigen (B19 VP1 protein) to that used in the immunoassay (B19 VP2 protein) making it an ideal standard reference test method for this purpose according to the National Committee for Clinical Laboratory Standards (NCCLS) guidelines. Parvovirus B19 PCR analysis was carried out essentially as described by Dieck et al. (1999). 


\subsection{Immunoglobulin class specificity}

Class specificity of the immunoassay was evaluated by testing specimens strongly reactive for both parvovirus $\mathrm{B} 19 \mathrm{IgG}$ and $\mathrm{IgM}$ in the parvovirus B19 IgM enzyme immunoassay as follows: parvovirus $\mathrm{B} 19 \mathrm{IgM}$ positive sera $(N=10)$ were tested (i) without absorbents as a control to demonstrate the reactive parvovirus B19 IgM status of the sera, (ii) IgG absorbed (goat anti-human IgG (Incstar, USA)) to show that IgG removal does not affect significantly the absorbance or index values relative to the control and therefore confirm IgG is not contributing to the signal, (iii) IgM absorbed (goat anti-human IgM (Dako)) to show that IgM removal eliminates the immunoassay signal thereby demonstrating absorbent efficacy and class specificity.

\subsection{Immunoassay reproducibility}

Intra-assay reproducibility was evaluated by testing a panel comprising parvovirus B19 IgM nonreactive, weakly and strongly reactive specimens $(N=12)$. Each specimen was assayed a total of 22 times. Inter-assay reproducibility data was determined as follows: a panel of coded parvovirus B19 IgM nonreactive, weakly reactive and reactive specimens $(N=16)$ was assayed across three independent batches of the immunoassay at three test sites over 3 days. Each specimen was assayed three times per day (in duplicate) per batch on 3 different days at each of three laboratories $(N=81$ assays per specimen). The test panel comprised nonreactive parvovirus B19 IgM (serum $N=2$, heparinised plasma $N=1$, EDTA plasma $N=1$ ), weakly reactive parvovirus B19 IgM (serum $N=5$, heparinised plasma $N=1$, EDTA plasma $N=1$ ) and strongly reactive parvovirus $\mathrm{B} 19 \operatorname{IgM}$ (serum $N=3$, heparinised plasma $N=1$, EDTA plasma $N=1$ ) specimens. All weakly reactive specimens were prepared by diluting parvovirus B19 IgM reactive specimens into nonreactive specimens. Linear regression analysis was used to establish inter-laboratory $(N=3)$ and inter-batch $(N=3)$ reproducibility.

\subsection{Parvovirus B19 IgM seroprevalence}

The seroprevalence of parvovirus B19 $\operatorname{IgM}$ in a US blood donor population $(N=399)$ was determined using the parvovirus B19 IgM enzyme immunoassay. Here, consecutive healthy donors from two distinct locations in the USA (Houston $(N=200)$ and Minneapolis $(N=199))$ were included and both age and gender criteria were recorded. An aliquot of serum was taken from each eligible blood donor and then tested for parvovirus B19 IgM at the site of donation. There was no record of a parvovirus B19 epidemic or outbreak at the time of specimen collection.

\section{Results}

The relative sensitivity and specificity of the parvovirus B19 IgM detection at various immunoassay cut-off values is given in Fig. 1 (ROC plot for parvovirus B19 IgM detection). The actual immunoassay cut-off (1.0) results in a immunoassay sensitivity and specificity of 89.1 and $99.4 \%$, respectively. It is clear from this data that immunoassay sensitivity increases with decreasing cut-off value with a concomitant decrease in specificity. The clinical accuracy of the immunoassay is evident given the position of the selected cut-off in the upper left-hand quadrant of the plot.

Of the 110 parvovirus B19 clinical specimens tested to establish immunoassay sensitivity, 99

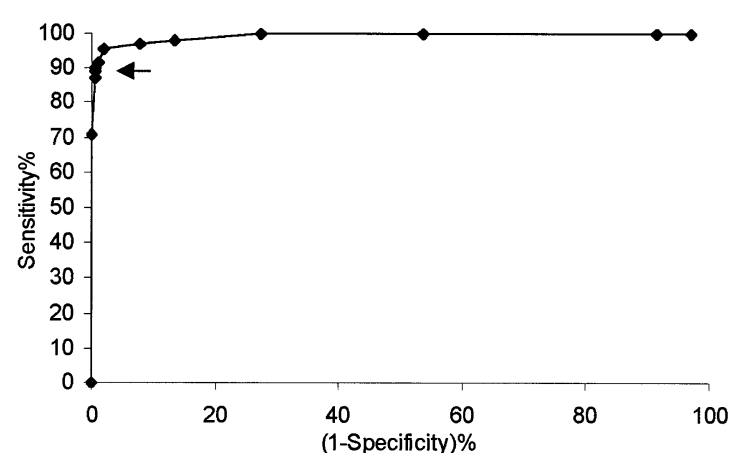

Fig. 1. ROC plot of the parvovirus B19 IgM enzyme immunoassay. The selected cut-off value is indicated with an arrow. 
Table 1

Parvovirus B19 IgM enzyme immunoassay specificity

\begin{tabular}{lll}
\hline Specimen Type & $\begin{array}{l}\text { No. of } \\
\text { Specimens }\end{array}$ & Number reactive \\
& & \\
\hline Cytomegalovirus & 21 & $0 / 21$ \\
EBV & 25 & $1 / 25^{\mathrm{b}}$ \\
Rubella & 20 & $0 / 20$ \\
Rubeola & 5 & $0 / 5$ \\
Mumps & 5 & $0 / 5$ \\
Varicella zoster virus & 5 & $0 / 5$ \\
Herpes simplex virus- 1 & 5 & $0 / 5$ \\
Herpes simplex virus-2 & 5 & $0 / 5$ \\
Parainfluenza 3 & 5 & $0 / 5$ \\
Influenza B & 4 & $0 / 4$ \\
Mycoplasma & 5 & $0 / 5^{\mathrm{c}}$ \\
Toxoplasma gondii & 14 & $0 / 14$ \\
Lyme disease & 5 & $0 / 5$ \\
Rheumatoid factor & 17 & $0 / 17$ \\
Rheumatoid arthritis & 5 & $0 / 5$ \\
Thyroiditis & 5 & $0 / 5$ \\
Systemic lupus & 5 & $0 / 5$ \\
$\quad$ erythematosus & & \\
Anti-nuclear antibody & 8 & $2 / 8^{*}$ \\
\hline
\end{tabular}

a Evaluation of specimens $(N=164)$ from patients with specific viral disease states or parvovirus B19 infection-like symptoms.

${ }^{\mathrm{b}}$ One EBV IgM and two ANA specimens which were reactive in the parvovirus $\mathrm{B} 19 \mathrm{IgM}$ enzyme immunoassay were also tested by parvovirus B19 immunoblot and immunofluorescent assays and were found to be reactive in both formats. Because the likelihood of genuine parvovirus B19 $\operatorname{IgM}$ reactivity cannot be eliminated they were excluded from the ROC analysis.

${ }^{\mathrm{c}}$ One mycoplasma specimen tested equivocal.

$(90 \%)$ were found to contain detectable parvovirus B19 IgM by immunoassay. The majority of the remaining specimens (7/11), gave index values greater than 0.6 which indicates the presence of very low levels parvovirus B19 IgM below the immunoassay cut-off value. A parvovirus B19 IgM IFA, which uses parvovirus B19 VP1 antigen, was used to determine parvovirus B19 IgM presence in 84 of the 110 clinical specimens. Three specimens which were reactive in the enzyme immunoassay were nonreactive by IFA and six nonreactive immunoassay specimens were weakly reactive by IFA.

Data presented in Table 1 shows immunoassay specificity when challenged with specimens from specific viral infection and disease states. No cross-reactivity was observed with a wide range of symptomatically similar viral infections including cytomegalovirus, rubella, mumps, VZV, rubeola, HSV-1 and 2. Of these 164 specimens used to evaluate immunoassay specificity for ROC analysis, only three exhibited apparent cross-reactivity whereby 1/21 EBV IgM specimens and 2/8 ANA specimens were reactive by the parvovirus B19 IgM immunoassay. Notably, the EBV IgM specimen and both ANA specimens were also reactive following parvovirus B19 IgM analysis by IFA and Western blot detection systems (data not shown). Insufficient EBV specimen volume was available for PCR analysis, however 1/2 ANA specimens was PCR positive. No reactivity was observed with all other disease states which may produce B19 infection-like symptoms and specimens which were from rheumatoid arthritis or lupus erythematosus patients, or contained rheumatoid factor, were nonreactive in the immunoassay (Table 1). Specimens obtained from toxoplasmosis $(N=14)$ and lyme disease infected individuals $(N=5)$ were also completely nonreactive in the parvovirus B19 IgM immunoassay. No evidence of significant cross-reactivity was detectable in mycoplasma, parainfluenza 3 and parainfluenza B IgM specimens tested by the parvovirus B19 IgM immunoassay, however 1/5 mycoplasma IgM specimens did give equivocal results on two occasions.

The parvovirus B19 IgM immunoassay is specific for parvovirus B19 IgM detection. Neither the presence or subsequent removal of parvovirus B19 IgG affects parvovirus B19 IgM analysis. Removal of total IgM from the ten parvovirus B19 IgM reactive specimens produces the expected negative result thereby confirming the immunoglobulin class specificity of the device.

Intra-assay reproducibility of the parvovirus B19 IgM enzyme immunoassay is extremely high and, expressed as percentage coefficient of variation $(\% \mathrm{CV})$, ranges from $8.2 \%$ for weakly reactive specimens to $5.1 \%$ for strongly reactive specimens, respectively (Table 2 ). Inter-assay reproducibility was extensively investigated whereby a blinded panel of specimens $(N=16)$ was tested using three batches of parvovirus B19 IgM immunoassay at each of three test sites (Table 3). All four nonreac- 
tive and five strongly reactive specimens, which were each tested a total of 81 times, were identified correctly on all occasions. Thus, $403(81 \times$ $5)$ and $324(81 \times 4)$ individual specimen analyses gave the expected result. The weakly reactive specimens $(N=7)$ which all produced index or optical density (OD) values proximal to the immunoassay cut-off were correctly identified in $542 / 567(95.5 \%)$ occasions. Thus, in a combined specimen analysis of 1294 tests, $98.1 \%$ of results were as expected.

Total inter-assay reproducibility (excluding data from nonreactive test specimens), ranges from 11.8 to $17.5 \% \mathrm{CV}$ or 18.0 to $29.5 \% \mathrm{CV}$ when expressed in terms of either index or OD values, respectively (Table 3). Linear regression analysis of inter-site data confirms the high reproducibility and robustness associated with the immunoassay and correlation coefficients of 0.99 were observed for both index and OD values, respectively, for both inter-site (site 1 vs. 2 , site 2 vs. 3 and 1 vs. 3 ) and inter-batch comparative scenarios.

The overall rate of parvovirus B19 IgM seropositivity is $1 \%(4 / 399)$. Results from each of the two population groups dispersed geographically show that the seroprevalence of parvovirus B19 $\operatorname{IgM}$ is low with rates of $0.5 \%(1 / 200)$ and

\section{Table 2}

Intra-assay reproducibility expressed in terms of OD on 22 replicates of each of 12 different serum specimens ranging in parvovirus B19 IgM levels from nonreactive to strongly reactive $^{\mathrm{a}}$

\begin{tabular}{lllll}
\hline Specimen & Mean OD & S.D. & $\% \mathrm{CV}$ & $N$ \\
\hline UR-K & 0.02 & 0.002 & 9.43 & 22 \\
UR-L & 0.03 & 0.002 & 3.91 & 22 \\
WR-D & 0.59 & 0.02 & 4.12 & 22 \\
WR-E & 0.46 & 0.02 & 4.73 & 22 \\
WR-F & 0.45 & 0.02 & 4.38 & 22 \\
WR-G & 0.45 & 0.037 & 8.20 & 22 \\
WR-H & 0.22 & 0.02 & 7.32 & 22 \\
WR-I & 0.29 & 0.01 & 5.16 & 22 \\
WR-J & 0.20 & 0.01 & 5.48 & 22 \\
SR-A & 1.46 & 0.074 & 5.10 & 22 \\
SR-B & 1.002 & 0.024 & 2.44 & 22 \\
SR-C & 0.99 & 0.02 & 1.87 & 22 \\
\hline
\end{tabular}

${ }^{\text {a }} \mathrm{OD}$, optical density $(450 / 620 \mathrm{~nm})$; index: ratio specimen/ cut-off value OD; SR, strong reactive; WR, weak reactive; UR, nonreactive.
Table 3

Inter-assay reproducibility of the parvovirus B19 IgM enzyme immunoassay $^{\mathrm{a}}$

\begin{tabular}{lllll}
\hline Specimen & Mean OD & S.D. & $\%$ CV $(\% C V$-index $)$ & $N$ \\
\hline UR-1S & 0.039 & 0.009 & $23.20(23.50)$ & 81 \\
UR-2S & 0.038 & 0.012 & $32.68(29.64)$ & 81 \\
UR-3E & 0.034 & 0.011 & $30.67(30.39)$ & 81 \\
UR-4H & 0.034 & 0.011 & $32.84(32.79)$ & 81 \\
WR-1S & 0.462 & 0.113 & $24.39(16.77)$ & 81 \\
WR-2S & 0.429 & 0.104 & $24.37(15.84)$ & 81 \\
WR-3E & 0.407 & 0.108 & $26.40(16.62)$ & 81 \\
WR-4S & 0.341 & 0.083 & $24.42(17.34)$ & 81 \\
WR-5S & 0.347 & 0.095 & $27.23(17.46)$ & 81 \\
WR-6S & 0.305 & 0.087 & $28.48(17.10)$ & 81 \\
WR-7H & 0.303 & 0.090 & $29.52(17.24)$ & 81 \\
SR-1S & 1.128 & 0.226 & $20.06(13.58)$ & 81 \\
SR-2E & 1.201 & 0.233 & $19.37(15.14)$ & 81 \\
SR-3H & 1.204 & 0.229 & $19.04(11.76)$ & 79 \\
SR-4S & 1.103 & 0.199 & $18.02(13.29)$ & 81 \\
SR-5S & 1.023 & 0.198 & $19.36(12.36)$ & 81
\end{tabular}

${ }^{\text {a }}$ Sixteen specimens ranging in Parvovirus B19 IgM levels from nonreactive to strongly reactive were tested. Data was accumulated over three test sites and three batches of parvovirus B19 IgM enzyme immunoassay over a 3-day period. OD, optical density $(450 / 620 \mathrm{~nm})$; index: ratio specimen/cutoff value OD; UR, nonreactive; WR, weakly reactive; SR, strong reactive; $\mathrm{S}$, serum; E, EDTA plasma; $\mathrm{H}$, heparinised plasma.

$1.5 \%$ (3/199) from Houston and Minneapolis, respectively. Of the 399 specimens tested, six specimens were either weakly reactive $(N=3)$ or equivocal $(N=3)$ after initial testing. Upon retesting of the three equivocal specimens, one remained equivocal, one tested negative and one was weakly reactive. Follow-up samples were not available to confirm the equivocal result. Consequently, 99\% (394/399) specimens were nonreactive for parvovirus B19 IgM, four were reactive and one was equivocal. The parvovirus B19 IgM serological data from the 399 US blood donors expressed in terms of index values (ratio of specimen/cut-off OD value) demonstrates that the majority of specimens from the healthy blood donors have index values which are well below the assay cut-off value. Only a small minority of samples $(N=5)$ have index values approaching the cut-off value.

The seroprevalence of parvovirus B19 IgM in women of child-bearing age was also evaluated in 
females from both Minneapolis and Houston $(N=181)$. The overall rate of positivity in this group was $1.65 \%(3 / 181)$ which was not significantly different from the reminder of the test population $(P=0.33$, Fischers Exact Test).

\section{Discussion}

The data presented represents the most extensive report to date of the performance of a parvovirus B19 IgM enzyme immunoassay available commercially. The report clearly indicates the very high specificity and sensitivity of the immunoassay and confirms that the selected cut-off value facilitates the accurate detection of parvovirus B19 IgM. ROC analysis is a powerful tool used to assess the clinical accuracy of laboratory tests (Zweig and Campbell, 1993; NCCLS, 1995). In this study, such an analysis was applied, for the first time, to the determination of the clinical accuracy of a parvovirus B19 IgM immunoassay. When plotted on a ROC curve, the resultant data clearly demonstrates that the parvovirus B19 IgM immunoassay provides a means of classifying parvovirus B19 IgM status (Fig. 1). The test comes very close to being optimal $(100 \%$ sensitivity and specificity is represented by inflection in the upper left-hand quadrant) insofar as the closer the plot comes to this point the greater the serological distinction between patients with parvovirus B19 infection and patients exhibiting similar symptomologies.

Using the current immunoassay cut-off, the observed specificity exceeds $99 \%$ and the sensitivity is almost $90 \%$ which provides maximum assay specificity thus ensuring accurate diagnosis of parvovirus B19 infection. Greater assay sensitivity could be obtained by reducing the cut-off value with a slight compromise on specificity. This may be of significance in the analysis of certain patient populations (e.g. immunocompromised individuals) for evidence of recent parvovirus B19 infection. The majority of specimens $(N=9 / 11)$ which tested parvovirus B19 IgM negative were obtained 4-15 months post-parvovirus B19 IgM seroconversion, thus the amount of antibody present may have been insufficient to be detected as a reactive specimen at the selected immunoassay cut-off. Five of these nine specimens were very weakly reactive by IFA, possibly reflecting a slightly elevated sensitivity of this assay format. It is our view that specificity of detection with regard to exposure to parvovirus B19 is more important than sensitivity of detection given the somewhat non-specific and sometimes confusing symptoms associated with parvovirus B19 infection (Jensen and Vestergaard, 1997). Indeed many other viral infections, particularly rubella, induce symptoms similar to parvovirus B19 (Thomas et al., 1999; Turner, 1999). However, for optimal assessment of the likelihood of infection and determination of the risk of fetal hydrops in pregnancy, serological analysis should also determine the parvovirus B19 IgG status of the patient and follow up specimens should be drawn from the patient, where possible (Searle et al., 1997).

The parvovirus B19 IgM immunoassay demonstrated no cross-reactivity whatsoever with sera from viral disease states such as rubella, CMV, VZV, mumps or rubeola, all of which could theoretically be confused with parvovirus B19 infection. This is highly significant since previous reports of parvovirus B19 immunoassays have nearly all reported significant cross-reactivity with rubella and CMV IgM (Sloots and Devine, 1996; Tolfvenstam et al., 1996). Of the 164 potentially cross-reactive specimens tested in this study, only 6 specimens were reactive when equivocal results are included as false positive. These $\operatorname{EBV}(N=2)$ and mycoplasma $(N=1)$ specimens which were reactive or equivocal in the immunoassay were also tested using a parvovirus B19 IgM IFA and Western blot assay (Kerr et al., 1999). The EBV specimens were found to be strongly reactive in both assays. These assays confirmed antibody reactivity with parvovirus B19 VP1 structural protein which is not present in the VP2-based parvovirus B19 IgM immunoassay. Thus, it is possible that EBV reactivation of lymphocytes capable of producing parvovirus $\mathrm{B} 19 \mathrm{IgM}$ is responsible for this apparent cross-reaction (Alpers et al., 1994). The mycoplasma sample which gave repeatedly equivocal results using the parvovirus B19 IgM immunoassay was nonreactive in both parvovirus B19 IgM IFA and Western blot, possi- 
bly because the level of antibody may have been too low to be detected by these methods or because of genuine cross-reactivity. The ANA positive sera which were reactive using the parvovirus B19 IgM immunoassay were also reactive when tested in the parvovirus B19 assays described above and one of the two ANA specimens was also PCR positive. This finding may be of clinical significance since parvovirus B19 has been implicated in the development of certain autoimmune diseases with associated the presence of ANA (Fawaz-Estrup, 1996). It is important to note that in the occasional cases of apparent false positivity, the samples exhibiting the highest titre for the specific infection (EBV and mycoplasma) or disease state (ANA) were not those which exhibited false positivity in the parvovirus B19 IgM immunoassay. This is further evidence that the mass or avidity of potential cross-reactants may not be responsible for the observed results and that genuine reactivity in the parvovirus B19 immunoassay may be present.

Previous studies on the performance evaluation of parvovirus B19 IgM immunoassays have given only very limited information with regard to immunoassay reproducibility (Sloots and Devine, 1996). The data presented above represents the most comprehensive report of the true intra-assay and inter-assay reproducibility, comprising interbatch and inter-site studies, of a parvovirus B19 IgM immunoassay to date. Intra-assay reproducibility data, whereby coefficients of variation range from 5.1 to $8.2 \%$, confirm not only the accurate coating of capture antibody [anti-human IgM] but also the extent to which individual immunoassay steps have been optimised (Table 2). Inter-assay reproducibility data has been generated from the analysis of 16 blinded specimens of various parvovirus $\mathrm{B} 19 \mathrm{IgM}$ reactivities at three test sites, incorporating three tests per day by different analysts, over a 3-day period using three batches of immunoassay (Table 3). Consequently, each specimen has been tested a total of 81 times. The resultant $\% \mathrm{CV}$ (minimum $11.8 \%$ for strongly reactive to maximum $17.5 \%$ for weakly reactive specimens) are highly acceptable especially when one considers that all strongly reactive and nonreactive specimens were diagnosed correctly. Weakly reactive specimens were detected correctly on $542 /$ $567(95.5 \%)$ of occasions and were either equivocal $\quad(N=19 / 567 ; \quad 3.35 \%) \quad$ or nonreactive $(N=6 / 567 ; 1.1 \%)$ in the remainder of cases. It is likely that a combination of close proximity to the immunoassay cut-off and minor non-homogeneity in specimen matrices during test panel preparation may have been responsible for this observation. A finding borne out by the fact that two specimens in particular (WR-6S and WR-7H), each with a mean index of 1.36 , were responsible for the majority $(N=21 / 25)$ of weakly reactive to equivocal/nonreactive fluctuations. The observed inter-site and inter-batch correlation data $\left(r^{2}=\right.$ 0.99 ) is particularly important as it serves to confirm maximum inter-batch consistency and the diagnostic accuracy of the parvovirus B19 IgM immunoassay in different laboratories. This is of particular significance given the general lack of regulatory requirements for parvovirus B19 antibody detection, particularly throughout the $\mathrm{Eu}-$ ropean Union.

The seroprevalence study demonstrates that the total rate of parvovirus $\mathrm{B} 19 \mathrm{IgM}$ positivity is $0.5 \%(1 / 200)$ and $1.5 \%(3 / 199)$, respectively, in two US populations distinct geographically with an overall rate of $1.0 \%$ parvovirus B19 IgM positivity observed when both populations are combined $(N=399)$. Notably, the reactive samples detected were either in or slightly above the equivocal range of the parvovirus B19 IgM immunoassay whereas nonreactive specimens are well displaced from the immunoassay cut-off. The majority of samples $(N=348)$ have index values less than 0.3 (mean index $=0.15$ ) which is well below the cut-off index of 1.00 .

The seroprevalence rate of $1.65 \%(3 / 181)$ observed in the sub-population of women of childbearing age was not significantly different to that in the remainder of the study population. In a similar study carried out in Europe using the parvovirus B19 IgM immunoassay, a population of Irish blood donors gave a parvovirus B19 IgM seroprevalence rate of $1 / 417(0.24 \%)$ or $0.72 \%$ if two equivocal test results were included as reactives (data not shown). In addition, Searle et al., (1997) have observed a parvovirus B19 IgM seroprevalence of $2.34 \%$ in cohort of 939 German 
women following exposure to, or suspicion of infection with, parvovirus B19 during pregnancy. Further studies using a larger population cohort may help clarify the significance of the observed difference in parvovirus B19 IgM seropositivity between the two groups in the present report.

In summary, data are presented which provide for the first time information both on the performance of a parvovirus B19 IgM enzyme immunoassay in terms of absolute cutoff standardisation-using ROC analysis, and a comprehensive multi-site evaluation of immunoassay reproducibility. This information will be of use to the clinical virologist in understanding the reliability of current parvovirus B19 serological assays in the absence of significant international standards for the performance criteria required for accurate detection of recent infection with parvovirus B19.

\section{References}

Alpers, C., Scheid, C., Bauer, G., 1994. Acute EBV infection results in IgM responses against unrelated viruses. Med. Micro. Lett. 3, 306-316.

Anderson, M.J., Higgins, P.G., Davis, L.R., Willman, L.S., Jones, S.E., Kidd, I.M., Pattison, J.R., Tyrrell, D.A.J., 1985. Experimental parvoviral infection in humans. J. Infect. Dis. 152 (2), 257-265.

Brown, C.S., Salimans, M.M.M., Noteborn, M.H.M., Harro, T.W., 1990. Antigenic parvovirus B19 coat proteins VP1 and VP2 produced in large quantities in a baculovirus expression system. Virus Res. 15, 197-212.

Brown, C.S., Van Lent, J.W.M., Vlak, J.M., Spaan, W.J.W., 1991. Assembly of empty capsids by using baculovirus recombinants expressing human parvovirus B19 structural proteins. J. Virol. 65, 2702-2706.

Cohen, B.J., 1997. Detection of parvovirus B19-specific IgM by antibody capture radioimmunoassay. J.Virol. Methods 66 (1), 1-4.

Dieck, D., Schild, R.L., Hansmann, M., Eis-Hubinger, A.M., 1999. Prenatal diagnosis of congenital parvovirus B19 infection: value of serological and PCR techniques in maternal and fetal serum. Prenat. Diagn. 19 (12), 1119-1123.

Fawaz-Estrup, F., 1996. Human parvovirus infection:rheumatoid manifestation, angioedemia, C1 esterase inhibitor deficency, ANA positivity and onset of systemic lupus erythematosis. J. Rheumatol. 23 (7), 1180-1185.

Kurtzman, G.J., Ozawa, K., Cohen, B., Hanson, G., Oseas, R., Blaese, R.M., Young, N.S., 1987. Chronic bone marrow failure due to persistant B19 parvovirus infection. N. Engl. J. Med. 317, 287-294.
Jensen, I.P., Vestergaard, B.F., 1997. Assessment of the specificity of a commercial human parvovirus B19 IgM assay. Clin. Diag. Virol. 7 (3), 133-137.

Jordan, J.A., 1996. Identification of human parvovirus B19 infection in idiopathic nonimmune hydrops fetalis. Am. J. Obstet. Gynecol. 174, 37-42.

Kerr, S., O'Keeffe, G., Kilty, C., Doyle, S., 1999. Undenatured parvovirus B19 antigens are necessary for the accurate serodiagnosis of parvovirus B19 IgG. J. Med. Virol. 52, $179-185$.

Mark, Y., Rogers, B.B., Oyer, C.E., 1993. Diagnosis and incidence of fetal parvovirus infection in an autopsy series. Ped. Pathol. 13, 381-386.

Morinet, F., D'Auriol, L., Tratschin, J.D., Galibert, F., 1989. Expression of human parvovirus B19 protein fused to protein A in Escherichia coli: recognition by $\operatorname{IgM}$ and $\mathrm{IgG}$ antibodies in human sera. J. Gen. Virol. 70, 3091-3097.

National Committee for Clinical Laboratory Standards (NCCLS), 1995. Assessment of the clinical accuracy of laboratory tests using receiver operating characteristic (ROC) plots; approved guideline. NCCLS document GP10-A, 15 (19), ISBN-1-56238-285-3.

O’Neill, H.J., Venugopal, K., Coyle, P.V., Gould, E.A., 1995. Development of an IgM capture assay for the diagnosis of B19 parvovirus infection using recombinant baculoviruses expressing VP1 or VP2 antigens. Clin. Diag. Virol. 3 (2), $181-190$.

Ozawa, K., Ayub, J., Yu-Shu, H., Kurtzman, G., Shimada, T., Young, N., 1987a. Novel transcription map for the B19 (human) pathogenic parvovirus. J. Virol. 61, 2395-2406.

Ozawa, K., Young, N., 1987b. Characterisation of capsid and noncapsid proteins of B19 parvovirus propagated in human erythroid bone marrow. J. Virol. 61 (8), 2627-2630.

Pickering, J.W., Forghani, B., Shell, G.R., Wu, L.X., 1998. Comparative evaluation of three recombinant antigenbased enzyme immunoassays for detection of $\operatorname{IgM}$ and $\mathrm{IgG}$ antibodies to human parvovirus B19. Clin. Diag. Virol. 9 (1), 57-63.

Sloots, T., Devine, P.L., 1996. Evaluation of four commercial enzyme immunoassays for detection of immunoglobulin $\mathbf{M}$ antibodies to human parvovirus B19. Eur. J. Clin. Micro. Infect. Dis. 15 (9), 758-761.

Searle, K., Guillard, C., Enders, G., 1997. Parvovirus B19 diagnosis in pregnant women- quantification of $\mathrm{IgG}$ antibody levels $(\mathrm{IU} / \mathrm{ml})$ with reference to the international parvovirus B19 standard serum. Infection 25 (1), 32-34.

Serjeant, G.R., Serjeant, G.E., Thomas, P.W., Anderson, M.J., Patou, G., Pattison, J.R., 1993. Human parvovirus infection in homozygous sickle cell disease. Lancet $34 \mathrm{i}$, 1237-1240.

Thomas, H.I., Barrett, E., Hesketh, L.M., Wynne, A., Morgan-Capner, P., 1999. Simultaneous IgM reactivity by EIA against more than one virus in measles, parvovirus B19 and rubella infection. J. Clin. Virol. 14 (2), 107-118.

Tolfvenstam, T., Ruden, U., Broliden, K., 1996. Evaluation of serological assays for identification of parvovirus B19 immunoglobulin M. Clin. Diag. Lab. Immunol. 3 (2), 147150. 
Török, T.J., 1997. Unusual clinical manifestations reported in patients with parvovirus B19 infection. In: Anderson, L.J., Young, N.S. (Eds.), Human Parvovirus B19, Monographs in Virology, vol. 20. Basel, Karger, pp. 6182.

Török, T.J., Wang, Q.Y., Gary, G.W., Yang, C.F., Finch, T.M., Anderson, L.J., 1992. Prenatal diagnosis of intrauterine parvovirus B19 infection by the polymerase chain reaction technique. Clin. Infect. Dis. 14, 149155.

Turner, A.J., 1999. Surveillance of congenital rubella in Great Britain. Rubella can be mistaken for parvovirus B19 infection. Br. Med. J. 319 (7201), 57-58.

Zweig, M.H., Campbell, G., 1993. Receiver-operating characteristic (ROC) plots: a fundamental evaluation tool in clinical medicine. Clin. Chem. 39, 561-577. 